\title{
PARAMETER VALUES EXCLUDED BY EXISTENCE CONDITIONS FOR BUOYANT DISSIPATIVE MOTIONS IN VERTICAL CHANNELS.*
}

\author{
BY \\ D. D. JOSEPH AND W. H. WARNER \\ Department of Aeronautics and Engineering Mechanics, University of Minnesota
}

\begin{abstract}
The nonexistence of steady, fully-developed solutions for frictionallyheated buoyant flow in vertical channels is established analytically. Explicit bounds on the values of parameters beyond which solutions to this nonlinear problem cannot exist are derived by integral estimation and by a priori comparison methods. The a priori bound shows that no solution exists if a linear combination of the pressure gradient, constant heat source and wall temperature difference is larger than a specific number proportional to the lowest eigenvalue of a linear comparison equation. The integral estimation procedure leads to the delineation of excluded regions in a parameter space; for parameter values in these regions, no solution exists. For boundary conditions permitting a symmetric solution, the integral estimates exclude further regions in the parameter space, while the power series solution leads to an excluded line of values.

1. Introduction. Becaused of the intricate structure of the Navier-Stokes equations, exact solutions may be deduced only for very restricted classes of possible fluid motions. Prominent among these are the fully-developed steady motions. These motions are popular candidates for exact mathematical analysis because they imply zero acceleration and hence do not involve the very difficult problems generated by inertial nonlinearities. Such solutions always involve material parameters, like viscosity; and, though it is frequently true that exact solutions of the Navier-Stokes equations exist for all values of the parameters, it is only for restricted values of the parameters that one may realize the postulated motion physically. This is the case with the simplest of the viscous fluid motions, Poiseuille and Couette flow. The vast literature on the stability of these motions is a consequence of the fact that the mathematical solutions which describe these flows are physically realizable only if the flow is stable against always present disturbances. Viewed in this way, the problem of stability is roughly equivalent to an examination of conditions under which particular mathematically possible fluid motions can be expected to occur in nature. The problem of stability is thus closely related to, and often identical with, the problem of uniqueness of solutions. The solution of this problem involves, in part, a delineation of the ranges of parameters for which the given motion may be expected to be stable (or uniquely determined by the given conditions).

Questions of stability and uniqueness, however, are not the only ones which bear upon the existence or nonexistence of some postulated motion. There are fully-developed motions, for example, which are mathematically possible when the parameters of the problem are restricted to certain ranges and not possible when the values of the parameters lie outside the restricted ranges. Here the direct question of existence re-
\end{abstract}

*Received July 22, 1966. The work of D. D. Joseph was supported in part by NASA grant (NGR24-005-065) to the Space Science Center of the University of Minnesota. 
places the stability-uniqueness question and leads, as in the latter, to the delineation of parameter ranges in which the postulated motion may or may not occur. The problems involved here are perhaps less familiar in fluid mechanics than their stability-uniqueness counterparts and our knowledge of them is considerably less developed.

The fully-developed pipe and channel flows are a case in point. If the material properties are taken as constant, then these motions are mathematically possible for unrestricted positive values of the relevant material parameters (e.g. Reynolds number). Of course not all the possible values are compatible with a stable motion. If, however, the problem is generalized to include the effects of frictional heating on the velocity distribution by way of thermally induced viscosity variations, then fullydeveloped solutions are possible, but possible only for a restricted range of the parameters and beyond this range the postulated solutions cannot exist (cf. Regirer [5], Kaganov [3], Joseph [1]). The difficulty here arises as a result of the coupling of the viscous dissipation with the temperature-dependent reciprocal of the viscosity in the energy equation. The energy equation is then of the type

$$
\nabla^{2} \psi+\lambda f(x, y) \phi(\psi)=0 .
$$

This equation, for the usual boundary conditions, has no solutions when $\lambda$ exceeds in value

$$
\Lambda \operatorname{Max}_{\psi \geq 0} \frac{\psi}{\phi(\psi)}
$$

where $\Lambda$ is the first eigenvalue of a linearized version of the given differential system (Joseph [2]). Here the viscosity reciprocal, or fluidity, $\phi(\psi)$ acts as a nonlinear heat source and the system is very different from the more familiar $\phi=$ const counterpart.

Another more involved case arises, and for essentially the same reason, in slightly compressible (temperature-dependent density) buoyant flow in channels. The phenomenon seems to have been noticed first by Ostrach, et al in a series of papers (see Ostrach [4] for a full discussion and references). Ostrach considered the plane, laminar, fully-developed flow of a "Boussinesq" fluid between heated vertical walls taking into account both buoyancy and viscous dissipation. For certain combinations of the (many) parameters of the problem, Ostrach found that his numerical procedures indicated the existence of either no solution or two solutions. It is our purpose here to examine this problem analytically, to examine the nonlinear structure responsible for this typical nonlinear behavior, to demonstrate the close analogy between this problem and the problem of frictionally heated flow of liquids mentioned earlier, to give a priori bounds on the values of parameters for which solutions do not exist and in general to develop a partial but mathematically rigorous treatment of the existence problem implied by this nonlinear system.

2. Mathematical formulation. There is no difficulty in the problem formulation. The geometry is simple: the flow is planar, say parallel to the $Y Z$-plane, between two vertical walls $Y= \pm a$, with the positive $Z$-axis in the upward vertical direction. Since the flow is to be "fully-developed," end conditions are unimportant, and the channel is taken as unbounded in the $Z$-direction. Zero velocity and given values for the temperature are prescribed at the walls. The equations are the usual continuity, momentum, and energy equations for steady, two-dimensional flow of a slightly compressible fluid; a buoyancy term $(-\rho g)$ appears on the "force" side of the $Z$-momentum equation and 
a heat source term $Q$ may be kept in the energy equation. The equation of state is taken to be

$$
\rho=\rho_{0}\left[1-\beta\left(T-T_{0}\right)\right],
$$

where $\rho_{0}$ is the density at the reference temperature $T_{0}$, which we will choose a little further on. All other properties- $\mu, \beta, k$, etc.-are taken as constants appropriate to the reference state.

If one eliminates the density $\rho$ by using the equation of state and keeps only lowestorder terms except in the buoyancy force, the resulting equations are those for a steady incompressible plane flow with density $\rho_{0}$. Both buoyancy terms $\left(-\rho_{0} g\right)$ and $\left(\rho_{0} \beta g\left(T-T_{0}\right)\right)$ are kept in the $Z$-momentum equation, though the first may be combined with the pressure gradient as a static head term. Finally, one looks for "fullydeveloped" flow solutions, i.e., those for which there is no cross-flow:

$$
V(Y, Z) \equiv 0 .
$$

Then the axial velocity $W$ must be a function of $Y$ only and the pressure $P$ must be a function of $Z$ only. One is then left with the $Z$-momentum equation and the energy equation governing $W(Y)$ and the temperature $T(Y, Z)$ (cf. Ostrach [4]).

In order to obtain physically meaningful solutions, we choose a constant pressure gradient, constant wall temperature, and take $T$ and $Q$ to be functions of $Y$ only ${ }^{1}$ :

$$
\begin{aligned}
T(-a, Z) & =T^{*}, \quad T(a, Z)=T^{* *}, \quad P^{\prime}(Z)=-P^{* *} ; \\
T(Y, Z) & =\tau(Y), \quad Q=Q(Y) .
\end{aligned}
$$

We assume $T^{*} \leq T^{* *}$, and choose the reference temperature $T_{0}$ to be equal to $T^{*}$. The pressure gradient and heat source are given quantities, as are the wall temperatures.

The postulated motion then must satisfy the requirements of the following boundaryvalue problem:

$$
\begin{gathered}
\mu\left(d^{2} W / d Y^{2}\right)+\rho_{0} \beta g\left[\tau(Y)-T^{*}\right]=\rho_{0} g-P^{* *} \equiv-P^{*}, \\
k\left(d^{2} \tau / d Y^{2}\right)+\mu(d W / d Y)^{2}+Q(Y)=0, \\
W( \pm a)=0 ; \quad \tau(-a)=T^{*} ; \tau(a)=T^{* *} .
\end{gathered}
$$

Here $P^{*}$ is a (constant) pressure gradient parameter based on variations from the static gradient $\rho_{0} g$. Positive $P^{*}$ signifies that the pressure drops vertically as $Z$ increases. We take $Q$ as constant and show that its effect, like that of the wall temperature difference, simulates the pressure gradient in driving the flow-not mechanically, but through the buoyancy mechanism.

It is convenient to put Eqs. (5) in dimensionless form. The nondimensionalization chosen is based on the fact that the interaction of the buoyancy and viscous dissipation terms is such that the boundary value problem is consistent only for a restricted range of parameters. Further, in order to show that the heat source and wall temperature diffcrence act like a pressure gradient, we change variables as follows:

${ }^{1}$ A linear variation in $Z$ may be kept in the temperature and pressure gradient terms, leading to the Rayleigh number effect studied by Ostrach [4]. Bounds can be found by similar, if more subtle, approaches in this case also. However, the presence of this parameter is not essential for the phenomenon under study, and we do not treat it here. 


$$
\begin{gathered}
Y=a y, \quad W(Y)=k u \cdot(Y) /\left(\rho_{0} \beta g a^{2}\right), \\
\tau(Y)=T^{*}+\frac{Q}{2 k}\left(a^{2}-Y^{2}\right)+\frac{T^{* *}-T^{*}}{2 a}(a+Y)+\mu k \psi(Y) /\left(\rho_{0} \beta g a^{2}\right)^{2} .
\end{gathered}
$$

Introduce new parameters defined by

$$
\begin{aligned}
p & =\rho_{0} \beta g a^{4} P^{*} / \mu k, \\
q & =\left(\rho_{0} \beta g a^{3}\right)^{2} Q / \mu k^{2}, \\
\psi^{*} & =\left(\rho_{0} \beta g a^{2}\right)^{2}\left(T^{* *}-T^{*}\right) / \mu k ;
\end{aligned}
$$

then Eqs. (5) become

$$
\begin{gathered}
d^{2} w / d y^{2}+\psi=-p-\psi^{*}(1+y) / 2-q\left(1-y^{2}\right) / 2, \\
d^{2} \psi / d y^{2}+(d w / d y)^{2}=0, \\
w( \pm 1)=\psi( \pm 1)=0 .
\end{gathered}
$$

The basic question we now ask is: Can values of $\left(p, \psi^{*}, q\right)$ be determined for which solutions of the boundary value problem (8) do not exist?

Split the problem into its symmetric and antisymmetric parts and treat the halfchannel $0 \leq y \leq 1$ as the basic region. Let

$$
\begin{aligned}
& 2 u(y)=w(y)+w(-y), \quad 2 v(y)=w(y)-w(-y), \\
& 2 \theta(y)=\psi(y)+\psi(-y), \quad 2 \sigma(y)=\psi(y)-\psi(-y) ;
\end{aligned}
$$

clearly, $u$ and $\theta$ are the even parts and $v$ and $\sigma$, the odd parts of $w$ and $\psi$, respectively. Equations (8) can then be rewritten:

$$
\begin{gathered}
u^{\prime \prime}+\theta=-\left(p+\psi^{*} / 2+q / 2\right)+q y^{2} / 2 \equiv-A+C y^{2}, \\
\theta^{\prime \prime}+u^{\prime 2}+v^{\prime 2}=0, \\
v^{\prime \prime}+\sigma=-\psi^{*} y / 2 \equiv-B y, \\
\sigma^{\prime \prime}+2 u^{\prime} v^{\prime}=0, \\
u^{\prime}(0)=\theta^{\prime}(0)=u(1)=\theta(1)=v(0)=\sigma(0)=v(1)=\sigma(1)=0 .
\end{gathered}
$$

Our attention will be directed primarily to the even parts of the solutions and to the first two of these equations. Some general properties of the solutions are easily derivable by examination of these equations, and we note them for later reference and use:

(a) No purely odd solution $w \equiv v, \psi \equiv \sigma$ is possible except the trivial one.

(b) A purely even solution $w \equiv u, \psi \equiv \theta$ corresponds to the "symmetric" case, i.e. $B=\psi^{*}=0, A=p+q / 2$, for equal wall temperatures.

(c) From Eq. (10b) and the boundary conditions on $\theta, \theta(y)$ is a convex, positive, monotone-decreasing function on $[0,1]$, with $\theta(0) \equiv \theta_{M}>0$ the maximum value of $\theta(y)$. We can, therefore, bound $\theta(y)$ using $\theta_{M}$ as a parameter:

$$
\theta_{M}(1-y) \leq \theta(y) \leq \theta_{M}, \quad 0 \leq y \leq 1 .
$$

(d) From Eq. (10a), we see that $u^{\prime \prime}$ will be of one sign if $-A+C y^{2}-\theta$ is of one sign. Since $\theta \geq 0$, we can conclude: 
(i) $u(y)$ is convex, positive, and monotone-decreasing from $u(0) \equiv u_{M}>0$ on $[0,1]$ if $A \geqq \max (C, 0)$;

(ii) $u(y)$ is concave, negative, and monotone-increasing from $u(0) \equiv-u_{m}$, $u_{m}>0$, on $[0,1]$ if $A \leq \min \left(C,-\theta_{M}\right)$.

With these preliminaries aside we can demonstrate that solutions of the boundary value problem (8) do not exist when the parameter values lie outside a restricted range. As a first element in this program we discuss

3. A priori bounds. Comparison methods are used here to establish a priori bounds on the parameters for the existence of solutions (cf. Joseph [2]). Consider the comparison problem

$$
\phi^{\prime \prime}+\lambda y^{2} \phi=0, \quad \phi^{\prime}(0)=\phi(1)=0
$$

Using the boundary conditions on $\phi$ and $\theta$, we have

$$
0=\int_{0}^{1}\left(\phi \theta^{\prime \prime}-\theta \phi^{\prime \prime}\right) d y=-\int_{0}^{1} \phi\left({u^{\prime 2}}^{2}+{v^{\prime}}^{2}\right) d y+\lambda \int_{0}^{1} y^{2} \theta \phi d y
$$

Therefore,

$$
\begin{aligned}
& \lambda^{-1}=\frac{\int_{0}^{1} y^{2} \theta \phi d y}{\int_{0}^{1} \phi\left(u^{\prime 2}+v^{\prime 2}\right) d y}=\frac{\int_{0}^{1}\left\{y^{2} \theta \phi\left({u^{\prime}}^{2}+{v^{\prime}}^{2}\right) /\left({u^{\prime}}^{2}+{v^{\prime}}^{2}\right)\right\} d y}{\int_{0}^{1} \phi\left({u^{\prime}}^{2}+{v^{\prime}}^{2}\right) d y} \\
& \leq \operatorname{Max}\left(y^{2} \theta /\left({u^{\prime}}^{2}+v^{\prime 2}\right)\right), \quad{u^{\prime 2}}^{2}+{v^{\prime 2}}^{2} \geq 0, \quad y_{\varepsilon}[0,1] \text {. }
\end{aligned}
$$

Since ${v^{\prime 2}}^{2} \geq 0$

$$
1 \leq \lambda \operatorname{Max}\left(y^{2} \theta / u^{\prime 2}\right), \quad u^{\prime 2} \geq 0
$$

Integrate Eq. (10a):

$$
u^{\prime}(y)_{-}=-A y+\frac{C y^{3}}{3}-\int_{0}^{\nu} \theta(\eta) d \eta .
$$

Since $\theta(y)$ is monotone-decreasing,

$$
\int_{0}^{\nu} \theta(\eta) d \eta \geq y \theta(y)
$$

and $y^{3} \leq y$ on $[0,1]$. Therefore, for the case $A>C>0$, we have

and

$$
\begin{aligned}
& u^{\prime}(y) \leq-A y+C y / 3-y \theta(y) \leq 0, \\
& u^{\prime 2}(y) \geq y^{2}\{A-C / 3+\theta(y)\}^{2}
\end{aligned}
$$

$$
1 \leq \lambda \operatorname{Max}\left(\theta /(A-C / 3+\theta)^{2}\right) .
$$

(For the case $A>0>C$ (negative heat source), simply drop the $C / 3$ term.) Thus, no matter what $\theta$ may be, we have the a priori bound

$$
1 \leq \lambda / 4(A-C / 3)
$$

or

$$
A-C / 3 \leq \lambda / 4
$$


Since one can show that the smallest value of $\lambda$ for the eigenvalue comparison problem (12) is the first root of

$$
J_{-1 / 4}\left(\lambda^{1 / 2} / 2\right)=0
$$

i.e.,

$$
\lambda^{1 / 2} / 2 \cong 2.0063, \quad \lambda \cong 16.101,
$$

we have:

Theonem I. No solution for the symmetric part of the problem (8), and hence for the whole problem, exists if

$$
A-C / 3=p+\psi^{*} / 2+q / 6>\lambda / 4 \cong 4.026
$$

where $\lambda$ is the first root of $J_{-1 / 4}\left(\lambda^{1 / 2} / 2\right)=0$. This result holds for $A>C>0$, i.e. for $q>0, p+\psi^{*} / 2>0$. If $q<0$, no solution exists if

$$
A=p+\psi^{*} / 2+q / 2>\lambda / 4 .
$$

We next examine the problem from another point of view and achieve a more detailed description of possible solution regions.

4. Integral bounds. The formal integral solutions of Eqs. (10a, b) are:

$$
\begin{aligned}
& u^{\prime}(y)=-A y+C y^{3} / 3-\int_{0}^{\nu} \theta(\eta) d \eta, \\
& u(y)=A\left(1-y^{2}\right) / 2-C\left(1-y^{4}\right) / 12+\int_{\nu}^{1} \int_{0}^{\rho} \theta(\eta) d \eta d \rho, \\
& \theta^{\prime}(y)=-\int_{0}^{\nu}\left({u^{\prime}}^{2}+{v^{\prime}}^{2}\right) d \eta, \\
& \theta(y)=\int_{\nu}^{1} \int_{0}^{\delta}\left({u^{\prime 2}}^{2}(\eta)+v^{\prime 2}(\eta)\right) d \eta d \delta .
\end{aligned}
$$

Since ${v^{\prime 2}}^{2}(y) \geq 0$,

$$
\theta(0)=\theta_{M} \geq \int_{0}^{1} \int_{0}^{\delta} u^{\prime 2}(\eta) d \eta d \delta
$$

with equality holding only in the symmetric case. It is a reasonably straightforward, if tedious, process now to produce a lower bound on the last integral, using the first of Eqs. (23). Since (Eq. (11))

$$
\theta_{M}(1-y) \leq \theta \leq \theta_{M},
$$

we have

$$
\theta_{M}\left(y-y^{2} / 2\right) \leq \int_{0}^{\nu} \theta(\eta) d \eta \leq y \theta_{M}
$$

and may show that

$$
\theta_{M}^{2} / 24 \leq \int_{0}^{1} \int_{0}^{\nu}\left(\int_{0}^{\delta} \theta(\eta) d \eta\right)^{2} d \delta d y \leq \theta_{M}^{2} / 12 .
$$


The first of Eqs. (23) gives us

$$
{u^{\prime 2}}^{2}=\left(A y-C y^{3} / 3\right)^{2}+2\left(A y-C y^{3} / 3\right) \int_{0}^{\nu} \theta(\eta) d \eta+\left(\int_{0}^{\nu} \theta(\eta) d \eta\right)^{2} .
$$

We distinguish three cases:

I. $A \geq \max (0, C / 3)$, which implies that $A y-C y^{3} / 3 \geq 0$ on $[0,1]$;

II. $A \leq \min (0, C / 3)$, which implies that $A y-C y^{3} / 3 \leq 0$ on $[0,1]$;

III. $\min (0, C / 3) \leq A \leq \max (0, C / 3)$, which implies that $A y-C y^{3} / 3$ has an internal zero at $y_{0}=(3 A / C)^{1 / 2}$ in $(0,1)$.

The third case will not be treated here; the algebra is difficult but not impossible. For Cases I and II, we can prove

Theorem II. Every solution of the boundary value problem (8) necessarily satisfies the following inequalities

(a) Case I: $A \geq \max (0, C / 3)$ :

$$
\theta_{M}: \geq \frac{1}{2520}\left(210 A^{2}-56 A C+5 C^{2}+294 A \theta_{M}-36 C \theta_{M}+105 \theta_{M}^{2}\right)
$$

(b) Case II: $A \leq \min (0, C / 3)$ :

$$
\theta_{M} \geq \frac{51}{2500}\left(210 A^{2}-56 A C+5 C^{2}+420 \theta_{M} A-56 C \theta_{M}+105 \theta_{M}^{2}\right)
$$

where $\theta_{M}$ is the maximum value of the symmetric part of the temperature distribution.

Proof. From (24) and (26), we have

$$
\begin{aligned}
\theta_{M} \geq \int_{0}^{1} \int_{0}^{\delta} u^{\prime 2}(\eta) d \eta d \delta=\int_{0}^{1} \int_{0}^{\delta}\left(A \eta-C \eta^{3} / 3\right)^{2} d \eta d \delta \\
\quad+2 \int_{0}^{1} \int_{0}^{\delta}\left[\left(A \eta-C \eta^{3} / 3\right) \int_{0}^{\eta} \theta(\xi) d \xi\right] d \eta d \delta+\int_{0}^{1} \int_{0}^{\delta}\left(\int_{0}^{\eta} \theta(\xi) d \xi\right)^{2} d \eta d \delta
\end{aligned}
$$

which we write as $\theta_{M} \geq I_{1}+I_{2}+I_{3}$.

The first integral, $I_{1}$, on the right may be evaluated directly:

$$
I_{1} \equiv \int_{0}^{1} \int_{0}^{\delta}\left(A \eta-C \eta^{3} / 3\right)^{2} d \eta d \delta=\frac{1}{25^{2} 2}\left(210 A^{2}-56 A C+5 C^{2}\right) .
$$

Equation (25b) provides a lower bound $\theta_{M}^{2} / 24 \equiv 105 \theta_{M}^{2} / 2520$ on the third integral $\mathrm{I}_{3}$. In Case I, $A \eta-C \eta^{3} / 3 \geq 0$, so that the lower bound in Eq. (25a), when multiplied by $\left(A \eta-C \eta^{3} / 3\right)$ and integrated, provides a lower bound on $I_{2}$ :

$$
I_{2} \geq 6 \theta_{M}(49 A-6 C) / 2520 \text {. }
$$

In Case II, $A \eta-C \eta^{3} / 3 \leq 0$, so that the upper bound in Eq. (25a) must be used; this provides the lower bound

$$
I_{2} \geq 28 \theta_{M}(15 A-2 C) / 2520 .
$$

Putting the lower bounds together, we obtain the relationships (27) and (28).

These relationships may be given a simple geometrical interpretation. Each may be written in the form $\mathcal{F}\left(A, C, \theta_{M}\right) \leq 0$, where the surfaces $\mathcal{F}=$ const are quadric surfaces in the parameter space $A, C, \theta_{M}$. Only those parts in the half-space $\theta_{M} \geq 0$ are of interest. The surface $\mathcal{F}=0$ divides the space into a region $\mathcal{F}>0$ where nonexistence of 
solution is assured and a region $\mathscr{F} \leq 0$ where the solution combinations-in particular, the solution surface $\theta_{M}=\theta_{M}(A, C)$-must lie. One may check, for example, that the surface $\mathcal{F}=0$ in Case $I$ is an ellipsoid, or rather that piece of the ellipsoid allowable under the Case I restrictions.

It is possible to construct a graphical representation of the results of Theorem II by looking at planar sections $C=$ constant. In particular, if we take $C=0$, we obtain the physically meaningful case of no heat source, as well as a particular section of the bounding surfaces. For $C=0$, there are only two cases, or rather Case III becomes $A=0$ and can be handled easily. The a priori bound is then

$$
A \leq \lambda / 4 \text {. }
$$

The bounds (27), (28) become

or

$$
\begin{array}{ll}
\theta_{M} \geq A^{2} / 12+7 A \theta_{M} / 60+\theta_{M}^{2} / 24, & A \geq 0 \\
\theta_{M} \geq A^{2} / 12+A \theta_{M} / 6+\theta_{M}^{2} / 24, & A \leq 0
\end{array}
$$

$$
\begin{aligned}
& \mathcal{F}\left(A, \theta_{M}\right) \equiv 10 A^{2}+14 A \theta_{M}+5 \theta_{M}^{2}-120 \theta_{M} \leq 0, \quad A \geq 0, \\
& \mathcal{G}\left(A, \theta_{M}\right) \equiv 10 A^{2}+20 A \theta_{M}+5 \theta_{M}^{2}-120 \theta_{M} \leq 0, \quad A \leq 0 .
\end{aligned}
$$

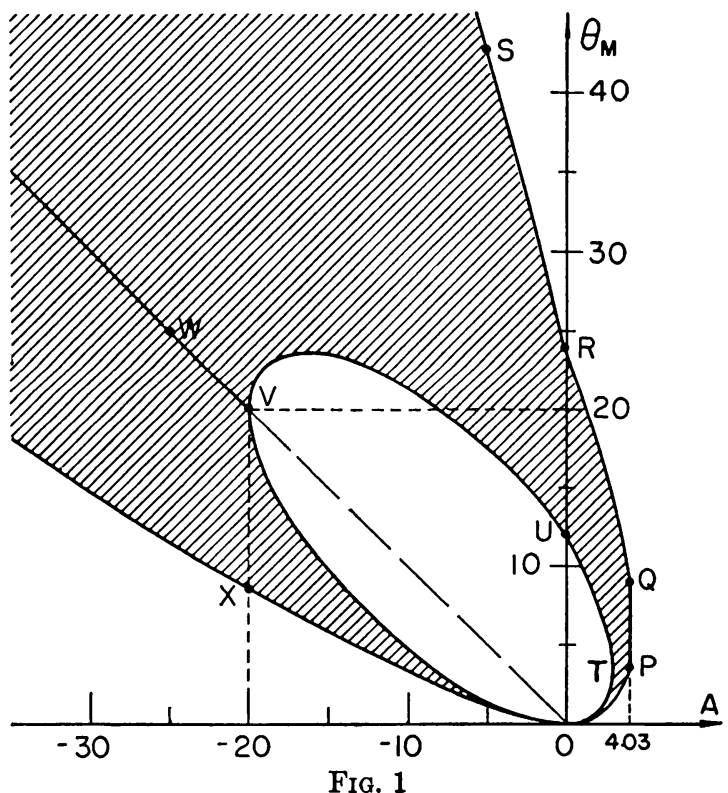

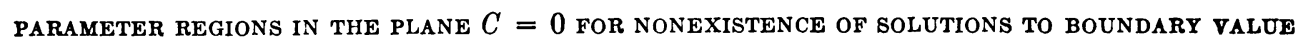
PROBLEM (8).

Unshaded regions, and the line VW, represent excluded combinations of $\left(A, \theta_{M}\right)$ determined by the conditions:

1. $\mathrm{PQ}$ : The a priori bound line (Eq. 21 , with $C=q=0$ )

2. OP, QR: Integral bound, Case I (Eq. 29)

3. RS, OX: Integral bound, Case II (Eq. 30)

4. OTU: Integral bound, symmetric problem, Case I, (Eq. 33)

5. OVU: Integral bound, symmetric problem, Case II, (Eq. 34)

6. VW: Series solution, symmetric problem 
The ellipse $\mathcal{F}=0$ and the hyperbola $\mathcal{G}=0$ are shown in Fig. 1 ; for values of $\left(A, \theta_{M}\right)$ "outside," no solution exists. The a priori bound $(A \cong 4.03)$ is also shown in Fig. 1 .

5. The symmetric problem. In the symmetric case $(B=0, A+p+q / 2=p+C)$, the result (24) is an equality, and we may also obtain upper bounds on $\theta_{M}$. In general, three cases must still be distinguished, as before. We treat only the first two.

Theorem III. If the wall temperatures are equal, every solution of the boundary value problem (8) necessarily satisfies the following inequalities:

(a) Case I: $A \geq \max (0, C / 3)$ :

$$
\theta_{M} \leq \frac{1}{2520}\left(210 A^{2}-56 A C+5 C^{2}+420 A \theta_{M}-56 C \theta_{M}+210 \theta_{M}^{2}\right) ;
$$

(b) Case II: $A \leq \min (0, C / 3)$ :

$$
\theta_{M} \leq \frac{1}{2520}\left(210 A^{2}-56 A C+5 C^{2}+294 A \theta_{M}-36 C \theta_{M}+210 \theta_{M}^{2}\right) .
$$

Here $\theta_{M} \equiv \psi_{M}$, the maximum value of the temperature distribution.

Proof. Follow Theorem II. Use of (24) (now an equality) and (26) results in

$$
\theta_{M}=I_{1}+I_{2}+I_{3} \text {, }
$$

where the integrals $I_{1}, I_{2}, I_{3}$ have the same meaning as in Theorem II. $I_{1}$ has the same value as before; $I_{3}$ is now bounded from above by $\theta_{M}^{2} / 12$, from Eq. (25b); and $I_{2}$ is bounded from above, using Eq. (25a), by interchanging the bounds for the two cases used in Theorem II. Relationships (31) and (32) result.

Again, the plane section $C=0$ permits a simple graphical representation. Physically, this means that the flow is driven by the pressure gradient and the frictionallyinduced density changes.

For $C=0$, the bounds become

$$
\begin{array}{ll}
\theta_{M} \leq A^{2} / 12+A \theta_{M} / 6+\theta_{M}^{2} / 12, & A \equiv p>0 ; \\
\theta_{M} \leq A^{2} / 12+7 A \theta_{M} / 60+\theta_{M}^{2} / 12, & A \equiv p<0,
\end{array}
$$

or

$$
\begin{aligned}
\mathcal{F}\left(A, \theta_{M}\right) & \equiv 10 A^{2}+20 A \theta_{M}+10 \theta_{M}^{2}-120 \theta_{M} \geq 0, \quad A \geq 0 ; \\
\mathscr{J}\left(A, \theta_{M}\right) & \equiv 10 A^{2}+14 A \theta_{M}+10 \theta_{M}^{2}-120 \theta_{M} \geq 0, \quad A \leq 0 .
\end{aligned}
$$

The parabola $\mathfrak{F C}=0$ and the ellipse $\mathscr{g}=0$ are shown in Fig. 1 also. Note that, for values of $\left(A, \theta_{M}\right)$ inside the oval made from piecing together $\Re C=0$ in the first quadrant and $\mathfrak{g}=0$ in the second, no solutions exist.

A final word on the integral bounds: Bounds on $u(0)$ can be obtained in a similar fashion, using (23). In the symmetric case, the case $A>0$ corresponds to a positive velocity field, with $u(0)=u_{M}$; the case $A<-\theta_{M}$ corresponds to downflow with $u(0)=-u_{m}$; and $-\theta_{M}<A<0$ corresponds to a velocity positive near the center but negative near the walls. The quantities $\theta_{M}$ and $u_{M}$ are of course not known, a priori. They may however be related to the given data by integral bounds on the equation $u(1)=0$. Finally we note that the value of other parameters, such as the value of the heat flux and shear stress at the wall, may be easily bounded by the above techniques.

Yet another limitation on possible parameter values in the symmetric, no heat source case may be deduced. With $w=u, \psi=\theta, A=p, B=C=0$, the power series solution to the equations can be written: 


$$
\begin{aligned}
& \theta=-A+\sum_{\nu=0}^{\infty} a_{\nu}\left(A+\theta_{M}\right)^{\nu+1} y^{4 \nu}, \\
& u=u(0)+\sum_{\nu=0}^{\infty} b_{\nu}\left(A+\theta_{M}\right)^{\nu+1} y^{4 \nu+2},
\end{aligned}
$$

where the coefficients are given by the recurrence relations

$$
\begin{gathered}
a_{0}=1, \quad a_{\nu}+(4 \nu+1)(4 \nu+2) b_{\nu}=0, \\
(4 \nu+3)(4 \nu+4) a_{\nu+1}+\sum_{r=0}^{n}(4 r+2)(4 \nu-4 r+2) b_{r} b_{\nu-r}=0 .
\end{gathered}
$$

The numbers $\theta_{M} \equiv \theta(0), u(0)$ are theoretically determined by the boundary conditions $\theta(1)=u(1)=0$. A number of interesting properties arise from investigation of these series. The one important to us here comes from setting $\theta_{M}=-A$. Then $\theta(y)=-A$, $u(y)=u(0)$, both constants; using the boundary conditions $\theta(1)=u(1)=0$, we see that the only solution possible for $\theta_{M}=-A$ occurs if $A=\theta_{M}=0$, i.e., if there is no flow or temperature variation.

Equivalently, we may examine the fourth-order differential equation system for our problem. Let $u=z_{1}(y), u^{\prime}=z_{2}(y), \theta=z_{3}(y), \theta^{\prime}=z_{4}(y)$; then we are interested in solving the problem

$$
\begin{gathered}
Z_{1}^{\prime}=Z_{2}, \quad Z_{2}^{\prime}=-A-Z_{3}, \quad Z_{3}^{\prime}=Z_{4}, \quad Z_{4}^{\prime}=-Z_{2}^{\prime 2}, \\
Z_{2}(0)=Z_{4}(0)=Z_{1}(1)=Z_{3}(1)=0 .
\end{gathered}
$$

The right-hand sides are analytic in $Z_{i}$, so that a unique trajectory exists through each point of phase space. The equilibrium points lie on the axis $\left(Z_{2}=Z_{4}=0, Z_{3}=-A\right)$ parallel to $Z_{1}$. Remembering that $Z_{3}(0)=\theta(0)=\theta_{M}$, we see that any solution starting with the initial value $\theta_{M}=-A$ must remain at the point $\left(Z_{1}(0), 0,-A, 0\right)$ at which it starts, by uniqueness. The only such solution satisfying the $Z_{1}(1)=Z_{3}(1)=0$ conditions is the $Z_{1}(0)=A=0$ solution: the 'no-flow' solution.

In Fig. 1, the line $\theta_{M}+A=0$ is shown. This line divides the region $A<0$ in two parts. It is possible, for a given $A$, that a temperature solution exists with initial value $\theta_{M}$ lying above the line or below the line. If this happens-as we suspect it must-the $\left(A, \theta_{M}\right)$ curves representing the double-valued solutions cannot intersect and the two branches must never cross the line $A+\theta_{M}=0$.

6. Concluding remarks. Without buoyancy or frictional heating the momentum and energy equations are uncoupled and may be solved separately. For these uncoupled cases solutions exist for unrestricted values of the parameters. It is the nonlinear coupling of buoyancy and viscous dissipation which implies a critical range of the parameters.

Without this coupling the parameter values are unrestricted; with it, restricted. It is in this sense that the construction of the a priori bound (21) is instructive. Here the calculation begins from the second order energy equation and the viscous dissipation $u^{\prime 2}$ may be regarded as a nonlinear functional of the temperature. The mathematical problem is then almost identical to the one mentioned in the introduction which governs the fully-developed flow of frictionally-heated liquids when that problem is generalized to include the effects of temperature-dependent viscosity. In both problems the dissipation function $\Phi$, which acts physically as a field source of heat, is a monotone func- 
tional of the temperature having the property that

$$
\theta / \Phi(\theta) \quad(\geq 0)
$$

is bounded. In the viscosity problem $\Phi$ is temperature-dependent through the viscosity. In the problem treated here $\Phi$ depends on the temperature implicitly through buoyancy effects on the velocity. The ultimate root of these buoyancy effects is again the temperature dependence of a material parameter, in this case the density.

\section{REFERENCES}

1. D. D. Joseph, Phys. Fluids 7, 1761 (1964)

2. D. D. Joseph, Quart. Appl. Math. 23, 349 (1966)

3. S. A. Kaganov, Intern. Chem. Eng. 3, 33 (1963)

4. S. Ostrach, Laminar flows with body forces, Theory of Laminar Flows, F. K. Moore, Ed., Princeton Univ. Press, Princeton, N. J. 1964

5. S. A. Regirer, Prikl. Math. Mekh., 22, 414 (1958) 